\title{
Light-Driven Droplet Manipulation Technologies for Lab-on-a-Chip Applications
}

\author{
Sung-Yong Park and Pei-Yu Chiou \\ Department of Mechanical and Aerospace Engineering, University of California, Los Angeles, CA 90095-1597, USA \\ Correspondence should be addressed to Pei-Yu Chiou, pychiou@seas.ucla.edu
}

Received 15 June 2011; Accepted 20 August 2011

Academic Editor: Aaron T. Ohta

Copyright ( $) 2011$ S.-Y. Park and P.-Y. Chiou. This is an open access article distributed under the Creative Commons Attribution License, which permits unrestricted use, distribution, and reproduction in any medium, provided the original work is properly cited.

Droplet-based (digital) microfluidics has been demonstrated in many lab-on-a-chip applications due to its free crosscontamination and no dispersion nature. Droplet manipulation mechanisms are versatile, and each has unique advantages and limitations. Recently, the idea of manipulating droplets with light beams either through optical forces or light-induced physical mechanisms has attracted some interests, since light can achieve 3D addressing, carry high energy density for high speed actuation, and be patterned and dynamically reconfigured to generate a large number of light beams for massively parallel manipulation. This paper reviews recent developments of various optical technologies for droplet manipulation and their applications in lab-on-achip.

\section{Introduction}

Microfluidic devices promise a broad range of biomedical and chemical applications due to their potentials of small volume requirement, short analysis and diagnostic time, high sensitivity, and high throughput analysis [1-3]. Recently, particular attention has been paid to droplet-based (digital) microfluidic devices since droplets isolated in immiscible oil or air can be free from cross-contamination and dispersion [4]. Numerous droplet-based applications including protein crystallization $[5,6]$, polymerase chain reaction (PCR) $[7$, $8]$, enzyme kinetic assays [3,9], and synthesis of organic molecules or nanoparticles $[10,11]$ have been demonstrated.

Droplet manipulation mechanisms that have been investigated cover a broad range of physical principles, including electrowetting on dielectric (EWOD) [12, 13], dielectrophoresis (DEP) $[14,15]$, thermocapillary force $[16,17]$, surface acoustic wave [18], magnetic force [19, 20], and optical forces [21]. In recent years, many light-driven droplet actuation mechanisms have been demonstrated, aiming to provide more functionalities, flexibility, lower cost, and higher throughput droplet manipulation tools or platforms $[22,23]$.
In general, optical-based droplet manipulation technologies can provide several unique advantages. First, light can be patterned and reconfigured to provide dynamic images, which in turn provides dynamic control of the triggered physical mechanisms without using complex control circuitry on chip. Millions of optical pixels can be readily generated by commercial spatial light modulators such as a LCD or DMD display to provide control of millions of electrodes in parallel on a low-cost and disposable device. Second, some optical methods can provide 3D manipulation of droplets since light can be propagated and focused in free space without needing any media. The energy can be delivered to any arbitrary locations in space to trigger an event.

This paper reviews optical manipulation mechanisms that have been utilized for manipulating droplets. A brief comparison of these technologies is summarized in Table 1 .

\section{Optical Droplet Manipulation Technologies}

Light-driven droplet manipulation technologies are in general based on three basic concepts: (1) direct optical forces, (2) opto-electrical, and (3) optothermal. Figure 1 shows 


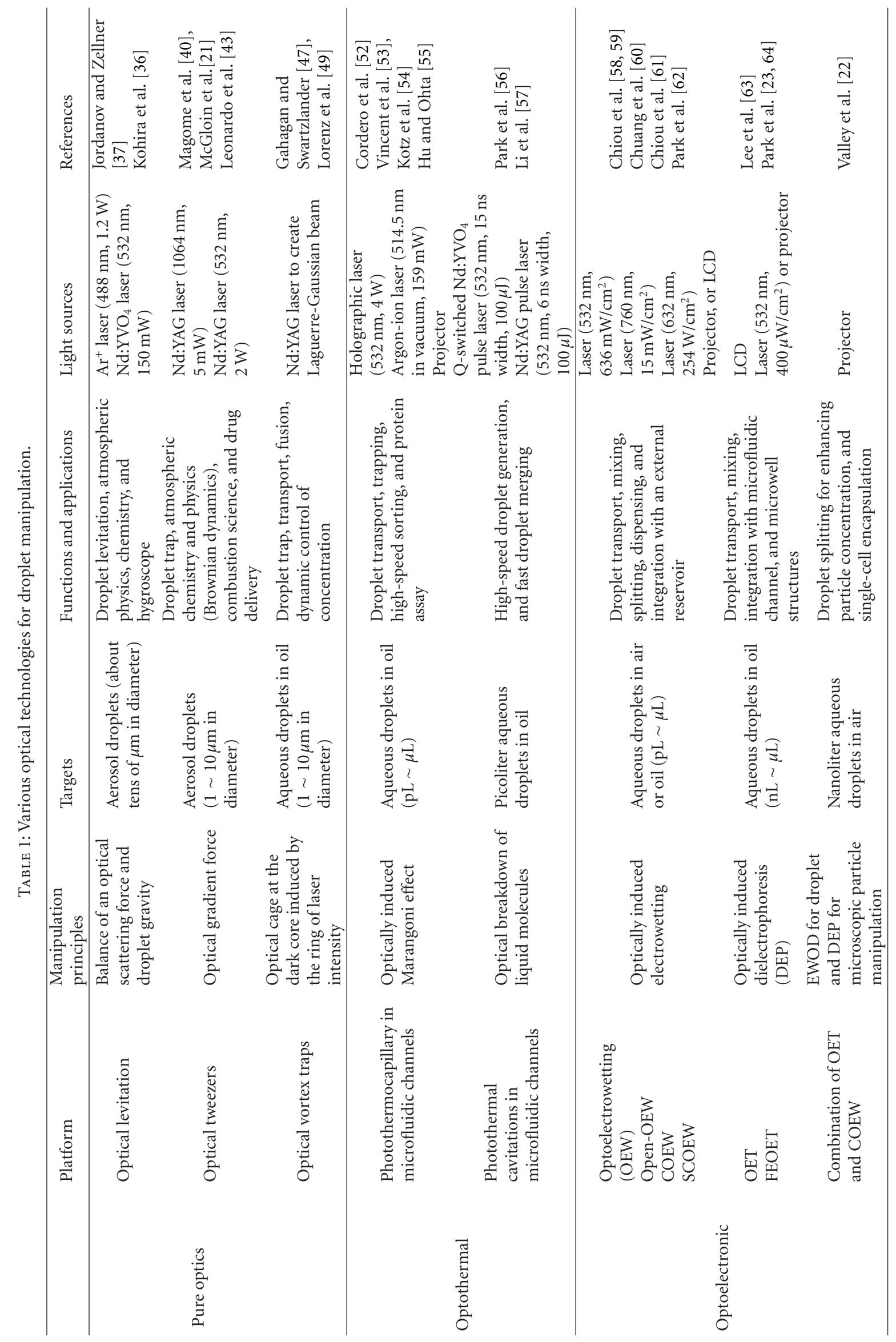




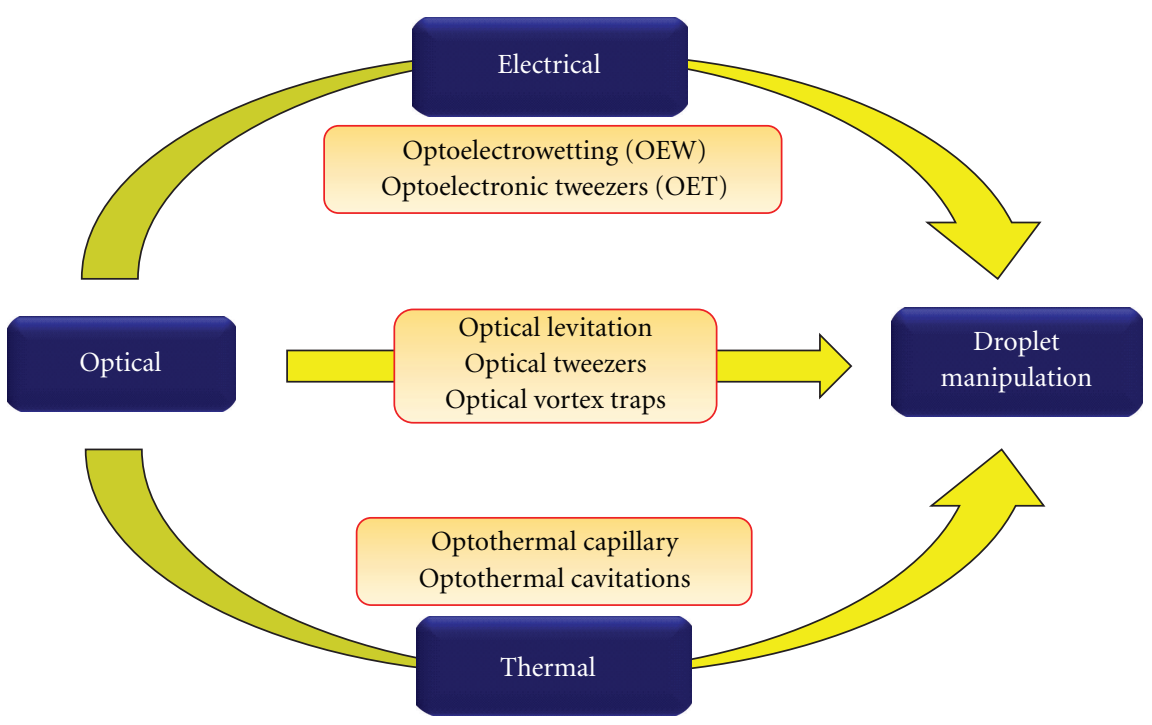

FIGURE 1: Illustration of optical energy transduction pathways used in optical methods for droplet manipulation.

the energy transduction pathways of mechanisms that have been applied for droplet manipulation. The principles, applications, and limitations of these mechanisms will be discussed in the following sections.

\subsection{Droplet Actuation Using Direct Optical Forces. Optical} tweezers is an useful technology in several fields of physics, chemistry, and biology [24]. It has been applied to trap, transport, pattern, and sort microscopic objects ranging from dielectric particles, viruses, living cells, bacteria, and small metal particles [24-31]. Two different types of forces are involved in optical manipulation. Scattering forces generate forces on particles in the light beam propagation direction [32], while gradient forces attract particles to regions of high intensity [24]. Since an optical force is typically in the range of $\mathrm{pN}$, only small size of droplets can be effectively manipulated.

2.1.1. Optical Levitation. Optical levitation is a method using a lightly focused light beam to irradiate an aerosol droplet and create a scattering force to balance the gravitation force of the droplet [33-35]. The size of suspended droplets is in the range of tens of micrometers. This technique has been used in measuring the droplet size and phase in thermodynamic equilibrium, which is controlled by temperature, composition of atmospheric aerosols, and relative humidity $[36,37]$.

2.1.2. Optical Tweezers and Optical Vortex Traps. A tightly focused laser beam is used to generate large gradient force to form a stable trap for an aerosol droplet smaller than $10 \mu \mathrm{m}$ in diameter, and prevent external perturbation from gas flow or collision of other aerosols [38]. The enhanced droplet position stability can provide excellent signal-to-noise ratio in data acquisition in short timescales. This gradient force of the optical tweezers can be expressed by [31]

$$
F=Q \frac{n P}{c}
$$

where $c / n$ is the light speed in the medium, $Q$ is the dimensionless trapping efficiency, and $P$ is the power of the incident light.

Figure 2 shows a typical experimental setup of an optical tweezers system for trapping dual aerosol droplets in gas [39]. An intense laser beam is focused through a high N.A objective lens to maximize the gradient force for optical trapping in a specially designed cell. Aerosols are generated from an ultrasonic nebulizer and introduced into the cell. The relative humidity is controlled by the flow rate of humidified nitrogen gas into the cell.

Magome et al. has demonstrated droplet trapping in air using optical tweezers [40]. The size and compositions of trapped droplets can be investigated using droplet cavity enhanced Raman spectroscopy (CERS) [41, 42]. This technique has shown that the droplet volume is preserved with extremely high precision when undergoing coagulation. Leonardo et al. have used optical trapping to study the Brownian dynamics of a water droplet, whose displacement is determined by the viscosity of the gas phase and the optical power for trapping [43]. Reid's group has studied extensively the relative humidity effects on the equilibrium droplet size, thermodynamic properties of an aerosol, chemical compositions, and coagulation using optical tweezers and Raman spectroscopy $[44,45]$. Holographic optical tweezers was also utilized to create multiple optical traps to manipulate an array of aerosol droplets in air and characterize the coagulation and mixing of multiple aerosols [21, 46].

Optical trapping using a strongly focused laser beam with a Gaussian profile allows trapping high refractive index objects $\left(n_{o}\right)$ in a low refractive index medium $\left(n_{m}\right)$ such as 


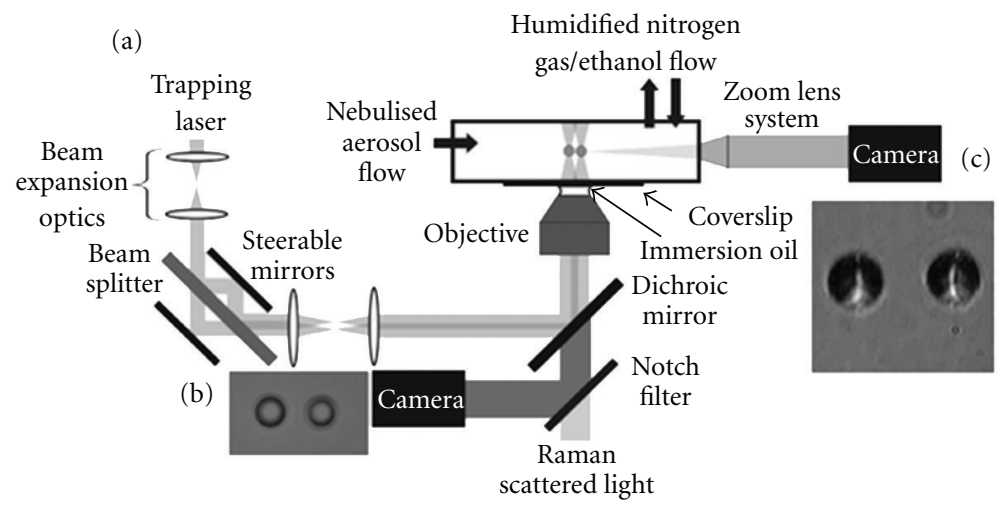

FIGURE 2: (a) Experimental setup of a dual trapping optical tweezers system. Reproduced with permission from [39] (b) In-plane image and (c) Side image of two optically trapped $\mathrm{NaCl} /$ aqueous aerosol droplets.

beads or cells dispersed in an aqueous buffer or aqueous droplets in air.

However, optical trapping of aqueous droplets dispersed in immiscible oil, which is a situation commonly used in digital microfluidics, cannot be achieved with this simple Gaussian beam, since the refractive index of water is lower than that of oil $\left(n_{\text {water }}<n_{\text {oil }}\right)$. The optical gradient force repels an oil-immersed water droplet, instead of trapping it.

To overcome this issue, Gahagan and Swartzlander have demonstrated three-dimensional trapping of low-index particles in water using a single dark optical vortex laser beam $[47,48]$. Instead of using Gaussian beam profile, optical vortex traps are based on the ring-shaped laser beam profile with a dark core in the focal point (Figure 3). Optical gradient force pushes a low-index particle away from the region of high light intensity. As a result, the ring-shaped laser beam forms a potential barrier and trap a low-index particle at the dark core, where a net force of all gradient forces is zero [49]. Compared to optical tweezers, optical vortex trapping provides several advantages. First, it enables a stable optical trap of low-index particles such as water droplets dispersed in oil. Second, the potential barrier created by an optical vortex trap allows isolating a single droplet from potential contamination from other droplets. Utilizing the optical vortex trapping technique, Chiu's group has demonstrated optical manipulation of femtoliter volume droplets immersed in oil such as droplet trap, fusion, and dynamic control of concentrations of dissolved species [50, 51].

2.2. Optothermal Droplet Actuation. Optics has been commonly used as heating sources in many biomedical and manufacturing areas [65]. Recently optothermal effects have been applied in manipulating droplets in digital microfluidics. Compared to direct optical forces, optothermal mechanisms could provide much larger forces to allow faster droplet manipulation in high-throughput applications.

2.2.1. Optothermal Capillary. The Marangoni effect is a phenomenon of liquid movement induced by surface tension difference. Thermocapillary is the Marangoni effect associated with surface tension difference induced by temperature gradient. This thermocapillary phenomenon was first investigated by Young et al. who observed air bubble motion in silicone oil induced by temperature gradient [66]. In an unconfined fluid, thermocapillary migration speed, $\mathbf{U}_{\mathrm{Th}}$, of a droplet can be expressed by [67]

$$
\mathbf{U}_{\mathrm{Th}}=-\frac{2}{2 \mu_{o}+3 \mu_{i}}\left(\frac{\partial \sigma}{\partial T}\right) \frac{R}{2+\Lambda_{i} / \Lambda_{o}} \nabla T,
$$

where $R$ is the droplet radius, $\mu_{o}$ and $\mu_{i}$ are the shear viscosity, and $\Lambda_{o}$ and $\Lambda_{i}$ the thermal conductivities of the fluid inside and outside the droplet. The thermocapillary migration speed is proportional to droplet size $(R)$, the ratio of change of surface tension with temperature $(\partial \sigma / \partial \mathrm{T})$, and temperature gradient $(\nabla \mathrm{T})$. It is noted that when $\partial \sigma / \partial \mathrm{T}<0$, the droplet is attracted to the light illumination region, the hot zone, while it is repelled if $\partial \sigma / \partial \mathrm{T}>0$.

Conventional thermocapillary devices typically use microfabricated electrical resistors to generate heat and temperature gradient for droplet transport, trapping, and sorting on trajectories of prepatterned structures $[68,69]$. Optothermal capillary induces the Marangoni effect by localized laser heating at a droplet interface. This increases the local temperature and decreases the surface tension at the heated site. The light-induced surface tension gradient causes a droplet to move toward the colder region [54]. Optically induced thermocapillary forces have been used for demonstrating many droplet-based microfluidic phenomena. Baroud et al. showed the interfacial flow induced by a thermocapillary force at the water/oil interface (Figure 4(a)) [70]. In the case of droplets carried by immiscible oil flow in microchannels $(\partial \sigma / \partial \mathrm{T}>0)$, laser focusing at the front of a droplet causes a thermocapillary force to balance against the drag force from the hydrodynamic oil flow [70]. In addition, shaping a laser beam with holographic technology allows multidroplet patterning and merging [52]. Vincent et al. showed high-speed sorting and 100\% sorting efficiency for droplets moving at speed up to $1.3 \mathrm{~cm} / \mathrm{s}$ in microfluidic devices [53]. 


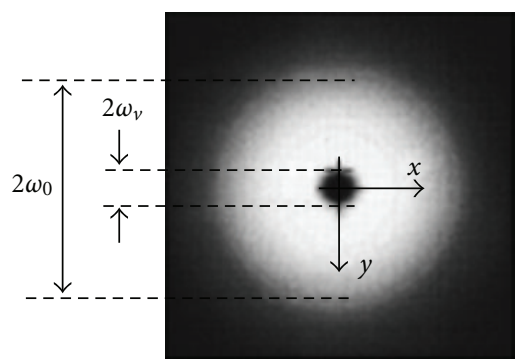

(a)

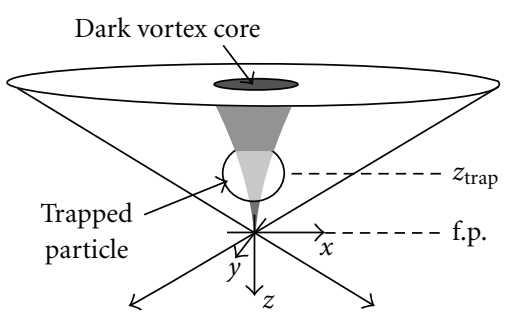

(b)

Figure 3: (a) Intensity profile of an optical vortex beam with beam waist $\omega_{0}$ and vortex waist $\omega_{v}$ at input aperture of focusing objective. (b) Schematic diagram of stable tapping for a low-index particle in an optical vortex trap. Reproduced with permission from [47].

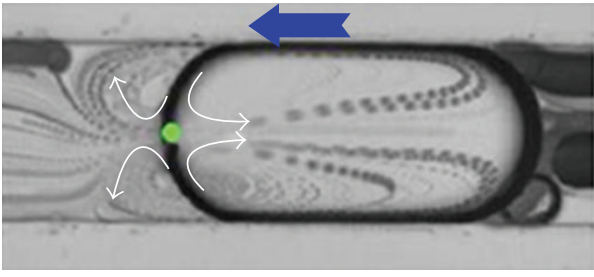

(a)

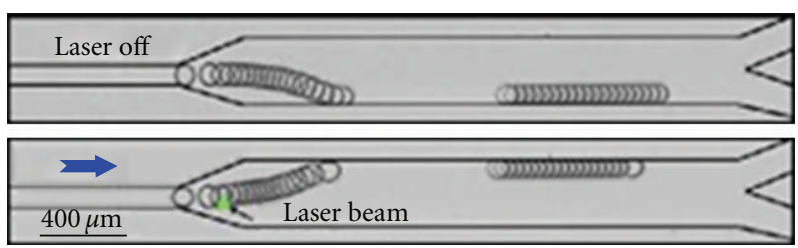

(b)

FIGURE 4: (a) Superposition of 100 frames from a video sequence showing the motion of seeding particles near the hot spot. Note that the motion along the interface is directed towards the hot spot. Reproduced with permission from [70]. (b) Superposition of successive frames illustrating drop switching by local thermocapillary actuation. The arrow indicates the laser location observed by the fluorescence of the water-dye solution. Reproduced with permission from [53].

Recently, Ohta et al. have also reported droplet manipulation driven by the optothermal capillary effect on a light absorbing a-Si:H coated glass substrate using an optical projector $[55,71]$.

2.2.2. Optothermal Cavitation Bubbles. Laser-induced cavitation is a phenomenon caused by tightly focusing an intense laser pulse in water to generate a rapidly expanding vapor bubble through nonlinear optical absorption [72]. Figure 5 illustrates the principle of laser-induced plasma formation followed by the emission of shock wave and the generation of a cavitation bubble. When a laser pulse is intensively focused into a liquid medium, such as water, strong optical fields can breakdown water molecules and produce hot plasma at the focal point (Figure 5(a)). This hot plasma leads to an explosive vapor bubble that expand at high speed and collapse rapidly (Figures 5(b) and 5(c)) [73-77]. The expansion speed of a laser-induced cavitation bubble is fast and can go up to hundreds of $\mathrm{m} / \mathrm{s}$ within $1 \mu \mathrm{s}$. Such high-speed cavitation bubbles have been used in many microfluidic applications such as cell lysing [77], microfluidic mixing [78], pumping [79], and switching [80].

Park et al. have demonstrated a high-speed, on-demand droplet generation device using such ultrafast microfluidic flow triggered by pulse laser induced cavitation bubbles. This mechanism is called pulse laser driven droplet generation (PLDG) [56]. It enables on-demand water droplet generation in microfluidic channels at a speed up to 10,000 droplets/sec with less than $1 \%$ droplet volume variation. PLDG devices have a simple structure, consisting of only two channels, one for water and one for oil, connected by a nozzle-shaped opening in a single-layer PDMS microfluidic chip (Figure 6). An intense laser pulse is focused in the middle of the water channel and induces a cavitation bubble to push water into the oil channel for droplet formation. By adjusting the laser energy and pulsing location, Park has demonstrated tunable droplet generation with volume ranging from $1 \mathrm{pL}$ to 150 pL. Recently, Li et al. also utilized laser induced cavitation bubbles to trigger on-demand droplet fusion in microfluidic devices [57].

2.3. Optoelectronic Droplet Actuation. Electrokinetics is one of the most commonly used methods for manipulating small-scale particles and microfluidic flows. Electrowetting on dielectric (EWOD) and dielectrophoresis (DEP) are the most effective electrical based mechanisms for droplet manipulation. DEP manipulates droplets by patterning nonuniform electric fields. Droplets do not necessarily contact the electrodes [82], while EWOD manipulates droplets by changing surface energy between droplets and the underneath dielectric material through electrostatic energy. It provides large actuation forces and fast responses [83, 84].

Optical control of electrokinetic phenomena is usually through photoconductive materials. Light illumination on a photoconductive layer can generate virtual electrodes to locally modify electric field distribution, which in turn controls the local DEP or EWOD effects for manipulating droplets. 

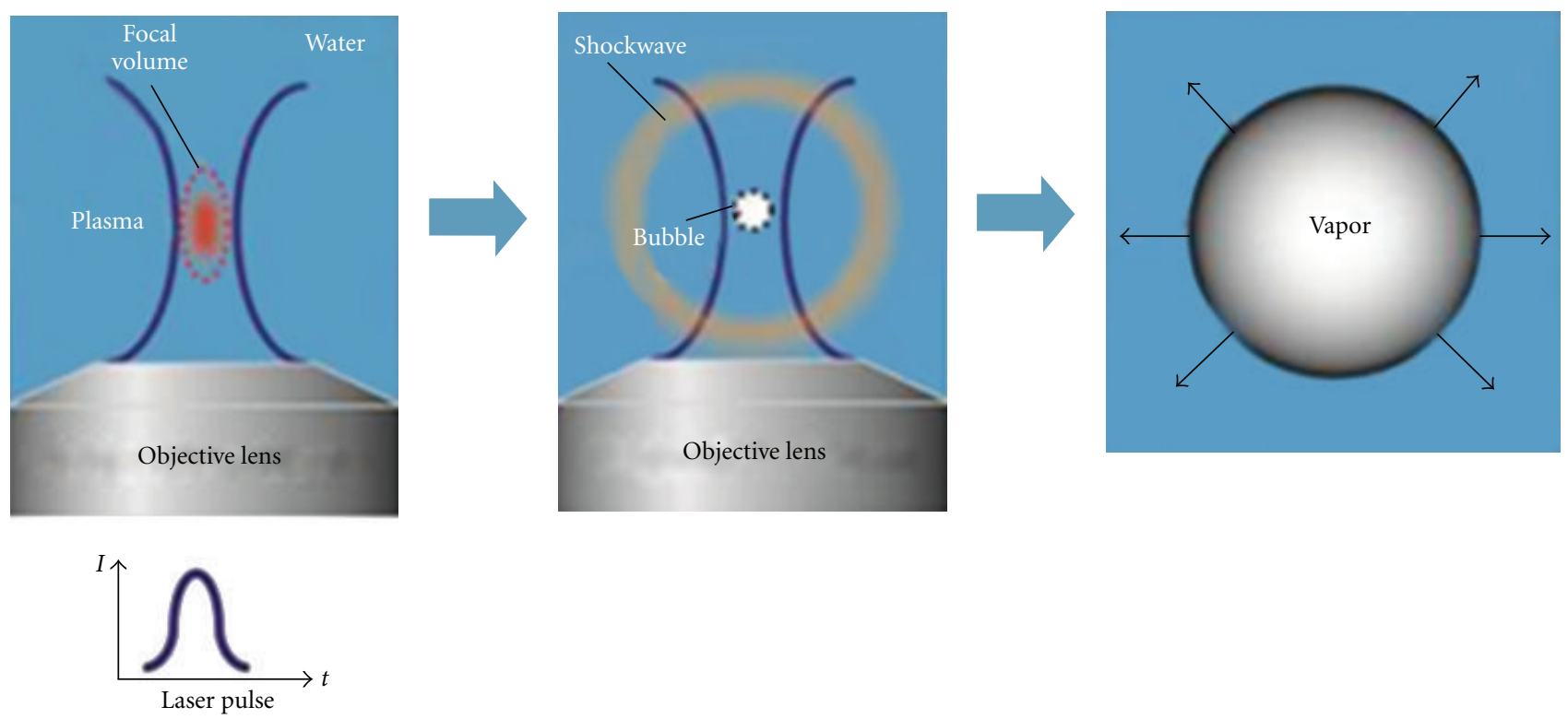

(a) Plasma formation

(b) Shockwave and bubble nucleation

(c) Bubble expansion and collapse

FIGURE 5: Schematic of pulse-laser induced plasma formation and the following shock wave emission and cavitation bubble generation in liquid medium. Reproduced with permission from [81].

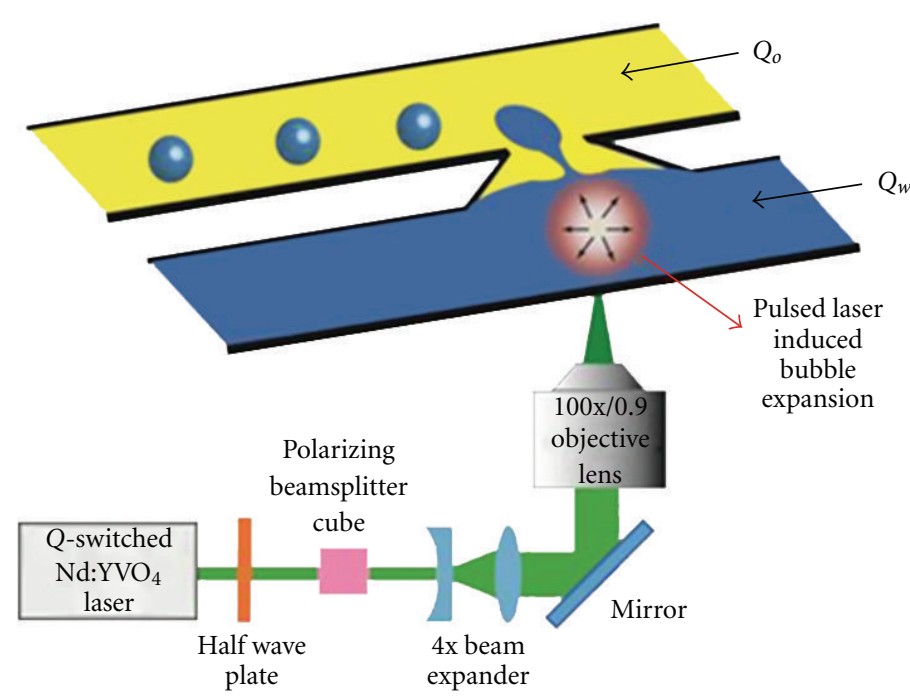

FIGURE 6: Schematic of a PLDG device that consists of two microfluidic channels connected by a nozzle-shape opening. A tightly focused laser pulse induces a rapid expanding bubble to push nearby water into the oil channel and form droplet. Reproduced with permission from [56].

2.3.1. Optoelecrowetting $(O E W)$. EWOD is an effective method for manipulating microdroplets. It utilizes electrostatic energy stored in the dielectric layer between a water droplet and an electrode to change local surface tension and droplet contract angle. The difference of contact angle at edges of a droplet induces a net capillary force to actuate the droplet [84]. EWOD has been shown to be able to provide a complete droplet functions from droplet injection, transportation, merging, mixing, and splitting [12, 85-87].

Young-Lippmann equation describes that the relationship between the contact angle $\theta$ of a droplet and the local voltage across a dielectric layer between the droplet and the underlying electrodes [88]

$$
\cos \theta=\cos \theta_{0}+\frac{1}{2 \gamma} \frac{\varepsilon_{r} \varepsilon_{0}}{t} V^{2},
$$

where $\theta_{0}$ is the original contact angle, $\gamma$ is the surface tension between the droplet and surrounding medium, $\varepsilon_{0}$ is vacuum permittivity, $\varepsilon_{r}$ is the dielectric constant, $t$ is the thickness of the dielectric layer, and $V$ is the voltage drop across a dielectric layer in the vertical direction at the three-phase contact line. 


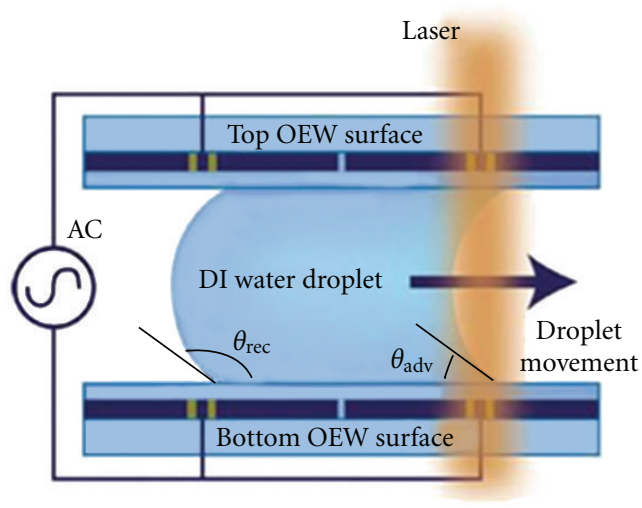

(a) Droplet transport

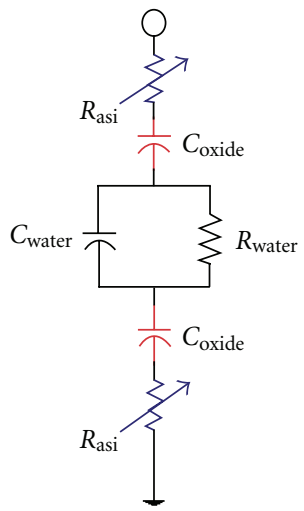

(b) Equivalent circuit

Figure 7: (a) Schematic of droplet actuation on an optoelectrowetting (OEW) device. (b) Its equivalent circuit model for one unit cell of OEW. Reproduced with permission from [58].

Droplet actuation on EWOD devices is typically realized by electrical activation of pixilated physical electrodes. Optoelectrowetting (OEW) uses optical images to trigger local electrowetting effects either on patterned digital electrodes or on a featureless photoconductive thin film. OEW solves the issues of complex wiring and interconnection when a large number of electrodes or droplets need to be controlled in parallel without interference.

The concept of OEW was first reported by Chiou et al. $[58,59]$. He demonstrated that all droplet manipulation functions including injection, transport, separation, and multidroplet manipulation could be achieved by light beam scanning on a photoconductive chip with tens of thousands of digital electrodes with only two electrical bias wires. Figure 7 shows the configuration of an OEW device and its equivalent circuit model of one unit cell. Each plate consists of patterned indium-tin-oxide (ITO) electrodes that are bridged by photoconductive hydrogenated amorphous silicon $(\mathrm{a}-\mathrm{Si}: \mathrm{H})$. Silicon dioxide $\left(\mathrm{SiO}_{2}\right)$ and thin Teflon layers are deposited to provide dielectric and hydrophobic properties on top of the ITO electrodes. An AC bias with frequency at $500 \mathrm{~Hz}$ is used for optimal operation and a light beam causes voltage switching between the oxide capacitors ( $\left.C_{\text {oxide }}\right)$ and the photoconductors $\left(R_{\text {asi }}\right)$. In the absence of light illumination, the electrical impedance of $R_{\text {asi }}$ dominates and most voltage drops across the photoconductive bridges. Therefore, ITO electrodes cannot be activated and droplet contact angle does not change. When light illuminates the photoconductive bridges nearby a droplet, the electric conductivity of the photoconductive bridges increases and ITO electrodes are activated to induce electrowetting for droplet actuation.

Conventional OEW configuration has two main drawbacks. First, it requires a sandwich structure consisting of two glass substrates. This increases the difficulty of interfacing with other microfluidic components. Second, minimum droplet size that can be actuated is limited by the size of digital electrodes as in regular EWOD devices. To overcome these limitations, several different OEW configurations have been proposed. Chuang et al. reported an open OEW configuration enabling droplet actuation on a single-sided photoconductive surface [60]. This open configuration provides a flexible interface to allow easy integration, but the patterned pixilated electrodes still limit the controllable minimum droplet size. Chiou et al. proposed a continuous optoelectrowetting (COEW) that allows continuous transport of picoliter droplets sandwiched between two featureless photoconductive electrodes [61], but it was faced with issues of device interfacing. Later Pei et al. reported the improved COEW device and showed more reliable fabrication that consequently enabled large array of manipulation of droplets [89].

Park et al. demonstrated a single-sided continuous optoelectrowetting mechanism (SCOEW) to enable continuous, light patterned electrowetting on a featureless photoconductive surface [62]. Unlike conventional OEW, droplet actuation on SCOEW is based on optical modulation of lateral electric fields. Under uniform light illumination, the contact angle of an oil-immersed water droplet remains larger than $90^{\circ}$ on top of the hydrophobic surface. As shown in Figure 8, the dark pattern illumination nearby the droplet locally increases the voltage drop across the dielectric layer. The decreased contact angle moves the droplet toward the dark region. SCOEW not only provides advantages of open configuration for easy integration and continuous manipulation to solve the size limitation issue but requires 3 orders of magnitude lower light intensity $\left(\sim 400 \mu \mathrm{W} / \mathrm{cm}^{2}\right)$ for droplet actuation than other OEW devices $\left(\sim 1 \mathrm{~W} / \mathrm{cm}^{2}\right)$. This unique advantage allows SCOEW to be operated by directly positioning a device on top of a LCD, an iPhone or an iPad screen without any extra light sources or lenses. One major limitation of SCOEW is that it requires high voltage (a few $\mathrm{kV}$ ) in actuation and optical control of droplet motion is less straightforward than conventional OEW.

2.3.2. Optoelectronic Tweezers (OET). Another widely applied principle for droplet manipulation is dielectrophoresis (DEP). It refers to an electrostatic force exerted on a 


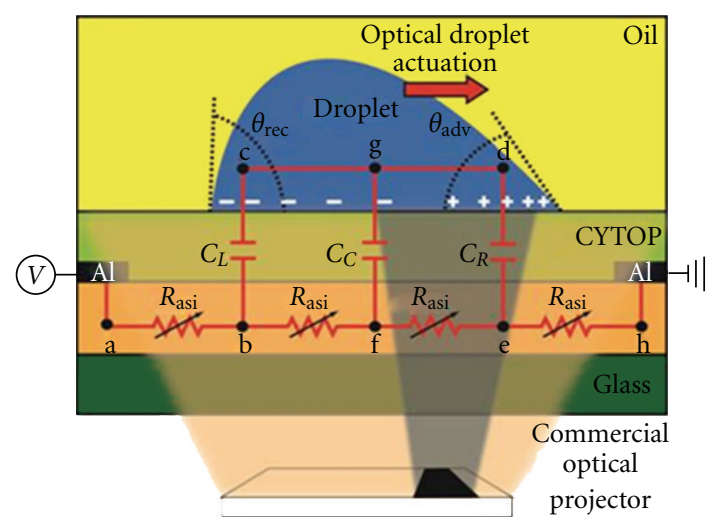

FIGURE 8: Schematic of a SCOEW device and its equivalent circuit model. Reproduced with permission from [62].

field induced electric dipole on a particle in a nonuniform electric field. This force can be approximated as [90]

$$
\mathbf{F}=(\mathbf{p} \cdot \nabla) \mathbf{E},
$$

where $\mathbf{p}$ represents the dipole moment and $\mathbf{E}$ is the electric field. For a homogenous dielectric sphere, the well-known expression for DEP force is given by [91]

$$
\begin{gathered}
\left\langle\mathbf{F}_{\mathrm{dep}}\right\rangle=2 \pi \varepsilon_{m} R^{3} \operatorname{Re}\left[K^{*}(\omega)\right] \nabla\left(\mathrm{E}_{\mathrm{rms}}{ }^{2}\right), \\
K^{*}(\omega)=\frac{\varepsilon_{p}^{*}-\varepsilon_{m}^{*}}{\varepsilon_{p}^{*}+2 \varepsilon_{m}^{*}}, \quad \varepsilon_{m}^{*}=\varepsilon_{m}+\frac{\sigma_{m}}{j \omega}, \varepsilon_{p}^{*}=\varepsilon_{p}+\frac{\sigma_{p}}{j \omega},
\end{gathered}
$$

where $\left\langle\mathbf{F}_{\text {dep }}\right\rangle$ represents the timeaverage of $\mathbf{F}_{\text {dep }}, \mathbf{E}_{\text {rms }}$ is the root-mean square magnitude of the electric field, $R$ is the particle radius, $\varepsilon_{m}$ and $\varepsilon_{p}$ are the permittivity of the surrounding medium and the particle, respectively, $\sigma_{m}$ and $\sigma_{p}$ are the electric conductivities of the medium and the particle, respectively, $\omega$ is the angular frequency of the applied electric field, and $K^{*}(\omega)$ is known as the frequency dependent Clausius-Mossotti (CM). The real part of $K^{*}(\omega)$ has a value, $0.5 \leq \operatorname{Re}\left[K^{*}(\omega)\right] \leq 1.0$, depending on the polarization of the medium and the particle at a certain frequency. In digital microfluidic devices where aqueous droplets are typically immersed in electrically insulating oil medium, $\operatorname{Re}\left[K^{*}(\omega)\right]$ has a positive value close to 1 , aqueous droplets in oli experience positive DEP forces that move them toward the strong electric field regions.

Chiou et al. have demonstrated a mechanism called optoelectronic tweezers (OET) to allow light images to pattern non-uniform electric fields to induce DEP forces for microparticle [92]. OET was commonly used for manipulating particles suspended in aqueous media. Due to an electrical impedance matching issue, OET is not ideal to operate in air or in oil environment since the large electrical impedance of these media will take over most voltage even without light illumination and optical modulation of electric field is difficult in principle. In some special cases in which droplet sizes are close to the gap space between two electrodes, optical actuation could still be accomplished. Park's group achieved droplet manipulation in oil using

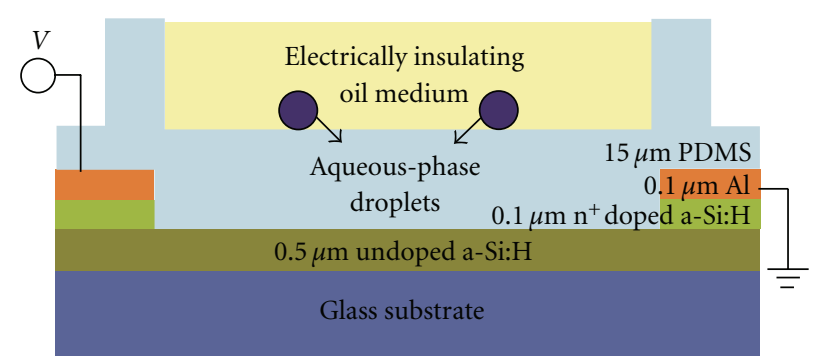

FIGURE 9: Schematic of a FEOET device. Reproduced with permission from [64].

a sandwiched OET platform integrated with microfluidic channels and showed the manipulation of picoliter droplets by optically induced DEP forces [63].

Park et al. have recently demonstrated a mechanism, called floating electrode optoelectronic tweezers (FEOET), specifically aiming for manipulating aqueous droplets suspended in electrically insulating oil and air media (Figure 9) [64]. FEOET utilizes lateral electric field as in SCOEW. The device structure of FEOET and SCOEW looks similar except that the thickness of the dielectric layer between droplets and the photoconductive layer is different. In SCOEW, this layer has to be as thin as $1 \mu \mathrm{m}$ to allow large contact angle modulation. In FEOET, this dielectric layer can be as thick as $1 \mathrm{~mm}$. The operation principle of FEOET is based the electrostatic interactions between a droplet induced dipole and the light induced dipole on the photoconductive layer under the application of a lateral field across the entire device. Both FEOET and SCOEW devices require extremely low light intensity in droplet actuation $\left(\sim 400 \mu \mathrm{W} / \mathrm{cm}^{2}\right)$ owing to the fact that in lateral electric field configuration, the virtual electrode is turned on as long as the light illumination can create a small photoconductivity difference between the illuminated and dark regions. This is different from other OET and OEW devices that require light to switch voltages between a photoconductor and a dielectric layer or a liquid layer. In other words, FEOET and SCOEW turn on virtual electrodes based on relative photoconductivity difference, not the absolute photoconductivity as in other devices. Using FEOET, Park has achieved transporting a $160-\mathrm{nL}$ droplet at a speed of $85.1 \mu \mathrm{m} / \mathrm{s}$ using a light beam with an intensity of $400 \mu \mathrm{W} / \mathrm{cm}^{2}$. Since FEOET allows a thick dielectric layer on top of the photoconductive surface, it provides a flexible interface for integration with other microfluidic components such as microwells and microchannels [23].

Recently, Valley et al. also reported an integrated platform enabling both COEW and OET manipulation on the same chip [22]. This platform allows light beams to manipulate not only droplets but also individual cells inside these droplets. They have demonstrated concentration enhancement of cells and single-cell encapsulation in droplet.

\section{Conclusion and Prospectus}

This paper reviews various optical manipulation methods that have been demonstrated so far for manipulating liquid 
droplets. These methods can be categorized into three types: (1) direct optical force, (2) optothermal, and (3) optoelectrical. Methods using direct optical forces can provide precise trapping of micrometer scale droplets in three dimensional spaces and are ideal tools for fundamental science studies. However, the high optical power requirement limits its throughput and applications in other areas.

Methods using optothermal effects can provide large forces for high-throughput and high-speed manipulation. Localized optical heating can generate temperature gradient to change surface tension, which is the dominating force in the micrometer scale, for droplet manipulation via the Marangoni effect. Optothermal actuation can also be accomplished by using intense short laser pulses to induce explosive cavitation bubbles which can generate large and transient pressure for high-speed microfluidic actuation. This novel actuation phenomenon has been utilized to demonstrate ondemand droplet injection up to 10,000 droplet/sec and ondemand droplet fusion.

Methods using optoelectrical effects could potentially provide a high throughput platform via massively parallel processing a large number of droplets across a large area. The major difference between optoelectrical from optothermal or direct optical force is that the optical energy is used to switch on electrical driving force to drive droplet motion, while in direct optical forces or in optothermal, the optical energy is directly used to power the droplet movement. This allows opto-electrical methods to actuate droplets with much lower optical power and induce minimum optical heating effect across a large area.

Optical methods for droplet manipulation are an emerging field that has already shown great promises for many applications. It provides several unique advantages that are difficult to achieve with other methods. It provides 3D addressing capability without the need of any physical electrical or mechanical interconnects that can be difficult to fabricate. Second, optical methods can deliver the highest energy density far beyond any other physical mechanisms can possibly achieve. If such energy can be properly converted into actuation forces through clever engineering designs, it can realize many novel high-speed droplet manipulation functions. Third, optical addressing provides a method to realize massively parallel droplet manipulation on a low cost and disposable platform.

Despite many promising potential applications for this technology, there are still many challenges that need to be overcome. First, many of the optical experiments still require bulky optics and lasers. Miniaturization of optical systems is critical to reduce footprint size of the integrated system, which is important for broadening future applications. A portable integrated system would be the goal. Most of the optical methods presented are enabling technologies that have to be integrated with other subsystems for real applications. Solving the interfacing issues will also be important. With the rapid progresses in photonics and optoelectronics, low-cost, compact, and high-power light sources, switches, and other components can be realized. More fundamental studies and research efforts need to be put on finding more novel and useful optical actuation mechanisms that have higher optical energy conversion efficiency to droplet manipulation, lower optical power requirement, more reliable devices and materials, and mechanisms that can be easily interfaced with other subsystems such as microfluidic devices.

\section{Acknowledgments}

This work is gratefully supported by an NSF CAREER Award (ECCS 0747950), NSF DBI-0852701, and NSF ECCS 0901154.

\section{References}

[1] H. Wang, K. Liu, K. J. Chen et al., "A rapid pathway toward a superb gene delivery system: programming structural and functional diversity into a supramolecular nanoparticle library," ACS Nano, vol. 4, no. 10, pp. 6235-6243, 2010.

[2] C. C. Lee, G. Sui, A. Elizarov et al., "Multistep synthesis of a radiolabeled imaging probe using integrated microfluidics," Science, vol. 310, no. 5755, pp. 1793-1796, 2005.

[3] L. S. Roach, H. Song, and R. F. Ismagilov, "Controlling nonspecific protein adsorption in a plug-based microfluidic system by controlling interfacial chemistry using fluorous-phase surfactants," Analytical Chemistry, vol. 77, no. 3, pp. 785-796, 2005.

[4] H. Song, D. L. Chen, and R. F. Ismagilov, "Reactions in droplets in microfluidic channels," Angewandte Chemie-International Edition, vol. 45, no. 44, pp. 7336-7356, 2006.

[5] B. T. C. Lau, C. A. Baitz, X. P. Dong, and C. L. Hansen, "A complete microfluidic screening platform for rational protein crystallization," Journal of the American Chemical Society, vol. 129, no. 3, pp. 454-455, 2007.

[6] D. L. Chen, G. J. Gerdts, and R. F. Ismagilov, "Using microfluidics to observe the effect of mixing on nucleation of protein crystals," Journal of the American Chemical Society, vol. 127, no. 27, pp. 9672-9673, 2005.

[7] W. Li, H. H. Pham, Z. Nie, B. MacDonald, A. Güenther, and E. Kumacheva, "Multi-step microfluidic polymerization reactions conducted in droplets: the internal trigger approach," Journal of the American Chemical Society, vol. 130, no. 30, pp. 9935-9941, 2008.

[8] Z. Hua, J. L. Rouse, A. E. Eckhardt et al., "Multiplexed realtime polymerase chain reaction on a digital microfluidic platform," Analytical Chemistry, vol. 82, no. 6, pp. 2310-2316, 2010.

[9] A. Huebner, M. Srisa-Art, D. Holt et al., "Quantitative detection of protein expression in single cells using droplet microfluidics," Chemical Communications, vol. 2, no. 12, pp. 1218-1220, 2007.

[10] L. H. Hung, K. M. Choi, W. Y. Tseng, Y. C. Tan, K. J. Shea, and A. P. Lee, "Alternating droplet generation and controlled dynamic droplet fusion in microfluidic device for CdS nanoparticle synthesis," Lab on a Chip, vol. 6, no. 2, pp. 174-178, 2006.

[11] T. Hatakeyama, D. L. Chen, and R. F. Ismagilov, "Microgramscale testing of reaction conditions in solution using nanoliter plugs in microfluidics with detection by MALDI-MS," Journal of the American Chemical Society, vol. 128, no. 8, pp. 25182519, 2006. 
[12] J. Gong and C. J. Kim, "All-electronic droplet generation on-chip with real-time feedback control for EWOD digital microfluidics," Lab on a Chip, vol. 8, no. 6, pp. 898-906, 2008.

[13] A. R. Wheeler, "Chemistry: putting electrowetting to work," Science, vol. 322, no. 5901, pp. 539-540, 2008.

[14] K. Ahn, C. Kerbage, T. P. Hunt, R. M. Westervelt, D. R. Link, and D. A. Weitz, "Dielectrophoretic manipulation of drops for high-speed microfluidic sorting devices," Applied Physics Letters, vol. 88, no. 2, Article ID 024104, pp. 1-3, 2006.

[15] J. R. Millman, K. H. Bhatt, B. G. Prevo, and O. D. Velev, "Anisotropic particle synthesis in dielectrophoretically controlled microdroplet reactors," Nature Materials, vol. 4, no. 1, pp. 98-102, 2005.

[16] J. Z. Chen, S. M. Troian, A. A. Darhuber, and S. Wagner, "Effect of contact angle hysteresis on thermocapillary droplet actuation," Journal of Applied Physics, vol. 97, no. 1, Article ID 014906, pp. 014906-9, 2005.

[17] E. F. Greco and R. O. Grigoriev, "Thermocapillary migration of interfacial droplets," Physics of Fluids, vol. 21, no. 4, Article ID 042105, 2009.

[18] T. Franke, A. R. Abate, D. A. Weitz, and A. Wixforth, "Surface acoustic wave (SAW) directed droplet flow in microfluidics for PDMS devices," Lab on a Chip, vol. 9, no. 18, pp. 2625-2627, 2009.

[19] M. Okochi, H. Tsuchiya, F. Kumazawa, M. Shikida, and H. Honda, "Droplet-based gene expression analysis using a device with magnetic force-based-droplet-handling system," Journal of Bioscience and Bioengineering, vol. 109, no. 2, pp. 193-197, 2009.

[20] Y. Zhang, S. Park, K. Liu, J. Tsuan, S. Yang, and T. H. Wang, "A surface topography assisted droplet manipulation platform for biomarker detection and pathogen identification," Lab on a Chip, vol. 11, no. 3, pp. 398-406, 2011.

[21] D. McGloin, D. R. Burnham, M. D. Summers, D. Rudd, N. Dewar, and S. Anand, "Optical manipulation of airborne particles: techniques and applications," Faraday Discussions, vol. 137, pp. 335-350, 2008.

[22] J. K. Valley, S. Ningpei, A. Jamshidi, H. Y. Hsu, and M. C. Wu, "A unified platform for optoelectrowetting and optoelectronic tweezers," Lab on a Chip, vol. 11, no. 7, pp. 1292-1297, 2011.

[23] S. Y. Park, S. Kalim, C. Callahan, M. A. Teitell, and E. P. Y. Chiou, "A light-induced dielectrophoretic droplet manipulation platform," Lab on a Chip, vol. 9, no. 22, pp. 3228-3235, 2009.

[24] A. Ashkin, J. M. Dziedzic, J. E. Bjorkholm, and S. Chu, "Observation of a single-beam gradient force optical trap for dielectric particles," Optics Letters, vol. 11, no. 5, pp. 288-290, 1986.

[25] J. E. Curtis, B. A. Koss, and D. G. Grier, "Dynamic holographic optical tweezers," Optics Communications, vol. 207, no. 1-6, pp. 169-175, 2002.

[26] K. Sasaki, M. Koshioka, H. Misawa, N. Kitamura, and H. Masuhara, "Optical trapping of a metal particle and a water droplet by a scanning laser beam," Applied Physics Letters, vol. 60, no. 7, pp. 807-809, 1992.

[27] A. Ashkin and J. M. Dziedzic, "Optical trapping and manipulation of viruses and bacteria," Science, vol. 235, no. 4795, pp. 1517-1520, 1987.

[28] A. Ashkin, J. M. Dziedzic, and T. Yamane, "Optical trapping and manipulation of single cells using infrared laser beams," Nature, vol. 330, no. 6150, pp. 769-771, 1987.

[29] D. G. Grier, "A revolution in optical manipulation," Nature, vol. 424, no. 6950, pp. 810-816, 2003.
[30] U. Bockelmann, P. Thomen, B. Essevaz-Roulet, V. Viasnoff, and F. Heslot, "Unzipping DNA with optical tweezers: high sequence sensitivity and force flips," Biophysical Journal, vol. 82, no. 3, pp. 1537-1553, 2002.

[31] J. E. Molloy and M. J. Padgett, "Lights, action: optical tweezers," Contemporary Physics, vol. 43, no. 4, pp. 241-258, 2002.

[32] A. Ashkin, "Acceleration and trapping of particles by radiation pressure," Physical Review Letters, vol. 24, no. 4, pp. 156-159, 1970.

[33] A. Ashkin and J. M. Dziedzic, "Optical levitation by radiation pressure," Applied Physics Letters, vol. 19, no. 8, pp. 283-285, 1971.

[34] A. Ashkin and J. M. Dziedzic, "Optical levitation of liquid drops by radiation pressure," Science, vol. 187, no. 4181, pp. 1073-1075, 1975.

[35] P. T. Nagy and G. P. Neitzel, "Optical levitation and transport of microdroplets: proof of concept," Physics of Fluids, vol. 20, no. 10, Article ID 101703, 2008.

[36] M. I. Kohira, A. Isomura, N. Magome, S. Mukai, and K. Yoshikawa, "Optical levitation of a droplet under a linear increase in gravitational acceleration," Chemical Physics Letters, vol. 414, no. 4-6, pp. 389-392, 2005.

[37] N. Jordanov and R. Zellner, "Investigations of the hygroscopic properties of ammonium sulfate and mixed ammonium sulfate and glutaric acid micro droplets by means of optical levitation and Raman spectroscopy," Physical Chemistry Chemical Physics, vol. 8, no. 23, pp. 2759-2764, 2006.

[38] R. J. Hopkins, L. Mitchem, A. D. Ward, and J. P. Reid, "Control and characterisation of a single aerosol droplet in a singlebeam gradient-force optical trap," Physical Chemistry Chemical Physics, vol. 6, no. 21, pp. 4924-4927, 2004.

[39] L. Mitchem, R. J. Hopkins, J. Buajarern, A. D. Ward, and J. P. Reid, "Comparative measurements of aerosol droplet growth," Chemical Physics Letters, vol. 432, no. 1-3, pp. 362-366, 2006.

[40] N. Magome, M. I. Kohira, E. Hayata, S. Mukai, and K. Yoshikawa, "Optical trapping of a growing water droplet in air," Journal of Physical Chemistry B, vol. 107, pp. 3988-3990, 2003.

[41] K. J. Knox, J. P. Reid, K. L. Hanford, A. J. Hudson, and L. Mitchem, "Direct measurements of the axial displacement and evolving size of optically trapped aerosol droplets," Journal of Optics A, vol. 9, no. 8, article S10, pp. S180-S188, 2007.

[42] R. Symes, R. M. Sayer, and J. P. Reid, "Cavity enhanced droplet spectroscopy: principles, perspectives and prospects," Physical Chemistry Chemical Physics, vol. 6, no. 3, pp. 474-487, 2004.

[43] R. D. Leonardo, G. Ruocco, J. Leach et al., "Parametric resonance of optically trapped aerosols," Physical Review Letters, vol. 99, no. 1, Article ID 010601, 2007.

[44] L. Mitchem, J. Buajarern, R. J. Hopkins et al., "Spectroscopy of growing and evaporating water droplets: exploring the variation in equilibrium droplet size with relative humidity," Journal of Physical Chemistry A, vol. 110, no. 26, pp. 8116$8125,2006$.

[45] L. Mitchem, J. Buajarern, A. D. Ward, and J. P. Reid, "A strategy for characterizing the mixing state of immiscible aerosol components and the formation of multiphase aerosol particles through coagulation," Journal of Physical Chemistry B, vol. 110, no. 28, pp. 13700-13703, 2006.

[46] D. R. Burnham and D. McGloin, "Holographic optical trapping of aerosol droplets," Optics Express, vol. 14, no. 9, pp. 4176-4181, 2006.

[47] K. T. Gahagan and G. A. Swartzlander, "Trapping of low-index microparticles in an optical vortex," Journal of the Optical Society of America B, vol. 15, no. 2, pp. 524-534, 1998. 
[48] K. T. Gahagan and G. A. Swartzlander, "Optical vortex trapping of particles," Optics Letters, vol. 21, no. 11, pp. 827-829, 1996.

[49] R. M. Lorenz, J. S. Edgar, G. D. M. Jeffries, Y. Zhao, D. McGloin, and D. T. Chiu, "Vortex-trap-induced fusion of femtoliter-volume aqueous droplets," Analytical Chemistry, vol. 79, no. 1, pp. 224-228, 2007.

[50] G. D. M. Jeffries, J. S. Kuo, and D. T. Chiu, "Dynamic modulation of chemical concentration in an aqueous droplet," Angewandte Chemie - International Edition, vol. 46, no. 8, pp. 1326-1328, 2007.

[51] D. T. Chiu and R. M. Lorenz, "Chemistry and biology in femtoliter and picoliter volume droplets," Accounts of Chemical Research, vol. 42, no. 5, pp. 649-658, 2009.

[52] M. L. Cordero, D. R. Burnham, C. N. Baroud, and D. McGloin, "Thermocapillary manipulation of droplets using holographic beam shaping: microfluidic pin ball," Applied Physics Letters, vol. 93, no. 3, Article ID 034107, 2008.

[53] M. R. D. S. Vincent, Ŕ. Wunenburger, and J. P. Delville, "Laser switching and sorting for high speed digital microfluidics," Applied Physics Letters, vol. 92, no. 15, Article ID 154105, 2008.

[54] K. T. Kotz, K. A. Noble, and G. W. Faris, "Optical microfluidics," Applied Physics Letters, vol. 85, no. 13, pp. 2658-2660, 2004.

[55] W. Hu and A. T. Ohta, "Aqueous droplet manipulation by optically induced Marangoni circulation," Microfluidics and Nanofluidics, vol. 11, no. 3, pp. 307-316, 2011.

[56] S. Y. Park, T. H. Wu, Y. Chen, M. A. Teitell, and P. Y. Chiou, "High-speed droplet generation on demand driven by pulse laser-induced cavitation," Lab on a Chip, vol. 11, no. 6, pp. 1010-1012, 2011.

[57] Z. G. Li, K. Ando, J. Q. Yu, A. Q. Liu, J. B. Zhang, and C. D. Ohl, "Fast on-demand droplet fusion using transient cavitation bubbles," Lab on a Chip, vol. 11, no. 11, pp. 1879-1885, 2011.

[58] P. Y. Chiou, Z. Chang, and M. C. Wu, "Droplet manipulation with light on optoelectrowetting device," Journal of Microelectromechanical Systems, vol. 17, no. 1, pp. 133-138, 2008.

[59] P. Y. Chiou, H. Moon, H. Toshiyoshi, C. J. Kim, and M. C. Wu, "Light actuation of liquid by optoelectrowetting," Sensors and Actuators A, vol. 104, no. 3, pp. 222-228, 2003.

[60] H. S. Chuang, A. Kumar, and S. T. Wereley, "Open optoelectrowetting droplet actuation," Applied Physics Letters, vol. 93, no. 6, Article ID 064104, 2008.

[61] P. Y. Chiou, S. Y. Park, and M. C. Wu, "Continuous optoelectrowetting for picoliter droplet manipulation," Applied Physics Letters, vol. 93, no. 22, Article ID 221110, 2008.

[62] S. Y. Park, M. A. Teitell, and E. P. Y. Chiou, "Singlesided continuous optoelectrowetting (SCOEW) for droplet manipulation with light patterns," Lab on a Chip, vol. 10, no. 13, pp. 1655-1661, 2010.

[63] D. H. Lee, H. Hwang, and J. K. Park, "Generation and manipulation of droplets in an optoelectrofluidic device integrated with microfluidic channels," Applied Physics Letters, vol. 95, no. 16, Article ID 164102, 2009.

[64] S. Park, C. Pan, T. H. Wu et al., "Floating electrode optoelectronic tweezers: light-driven dielectrophoretic droplet manipulation in electrically insulating oil medium," Applied Physics Letters, vol. 92, no. 15, Article ID 151101, 2008.

[65] L. J. Radziemski and D. A. Cremers, Laser-Induced Plasmas and Applications, Marcel Dekker, New York, NY, USA, 1989.

[66] N. O. Young, J. S. Goldstein, and M. J. Block, "The motion of bubbles in a vertical temperature gradient," Journal of Fluid Mechanics, vol. 6, pp. 350-356, 1959.
[67] K. D. Barton and R. S. Subramanian, "The migration of liquid drops in a vertical temperature gradient," Journal of Colloid and Interface Science, vol. 133, no. 1, pp. 211-222, 1989.

[68] B. Selva, V. Miralles, I. Cantat, and M. C. Jullien, "Thermocapillary actuation by optimized resistor pattern: bubbles and droplets displacing, switching and trapping," Lab on a Chip, vol. 10, no. 14, pp. 1835-1840, 2010.

[69] A. A. Darhuber, J. P. Valentino, J. M. Davis, S. M. Troian, and S. Wagner, "Microfluidic actuation by modulation of surface stresses," Applied Physics Letters, vol. 82, no. 4, pp. 657-659, 2003.

[70] C. N. Baroud, J. P. Delville, F. Gallaire, and R. Wunenburger, "Thermocapillary valve for droplet production and sorting," Physical Review E, vol. 75, no. 4, Article ID 046302, 2007.

[71] A. T. Ohta, A. Jamshidi, J. K. Valley, H. Y. Hsu, and M. C. Wu, "Optically actuated thermocapillary movement of gas bubbles on an absorbing substrate," Applied Physics Letters, vol. 91, no. 7, Article ID 074103, 2007.

[72] Y. R. Shen, The Principles of Nonlinear Optics, Wiley, New York, NY, USA, 1984.

[73] E. A. Brujan, K. Nahen, P. Schmidt, and A. Vogel, "Dynamics of laser-induced cavitation bubbles near an elastic boundary," Journal of Fluid Mechanics, vol. 433, pp. 251-281, 2001.

[74] R. K. Chang, J. H. Eickmans, W.-F. Hsieh, C. F. Wood, J.-Z. Zhang, and J.-b. Zheng, "Laser-induced breakdown in large transparent water droplets," Applied Optics, vol. 27, no. 12, pp. 2377-2385, 1988.

[75] A. Vogel, S. Busch, and U. Parlitz, "Shock wave emission and cavitation bubble generation by picosecond and nanosecond optical breakdown in water," Journal of the Acoustical Society of America, vol. 100, no. 1, pp. 148-165, 1996.

[76] E. Zwaan, S. Le Gac, K. Tsuji, and C. D. Ohl, "Controlled cavitation in microfluidic systems," Physical Review Letters, vol. 98, no. 25, Article ID 254501, 2007.

[77] K. R. Rau, P. A. Quinto-Su, A. N. Hellman, and V. Venugopalan, "Pulsed laser microbeam-induced cell lysis: timeresolved imaging and analysis of hydrodynamic effects," Biophysical Journal, vol. 91, no. 1, pp. 317-329, 2006.

[78] A. N. Hellman, K. R. Rau, H. H. Yoon et al., "Laser-induced mixing in microfluidic channels," Analytical Chemistry, vol. 79, no. 12, pp. 4484-4492, 2007.

[79] R. Dijkink and C. D. Ohl, "Laser-induced cavitation based micropump," Lab on a Chip, vol. 8, no. 10, pp. 1676-1681, 2008.

[80] T. H. Wu, L. Gao, Y. Chen, K. Wei, and P. Y. Chiou, "Pulsed laser triggered high speed microfluidic switch," Applied Physics Letters, vol. 93, no. 14, Article ID 144102, 2008.

[81] P.-Y. Chiou, T.-H. Wu, S. Park, and Y. Chen, "Pulse laser driven ultrafast micro and nanofluidic system," in Proceedings of the SPIE, vol. 7759, p. 77590Z, San Diego, Calif, USA, 2010.

[82] P. R. C. Gascoyne, J. V. Vykoukal, J. A. Schwartz et al., "Dielectrophoresis-based programmable fluidic processors," Lab on a Chip, vol. 4, no. 4, pp. 299-309, 2004.

[83] H. J. J. Verheijen and M. W. J. Prins, "Reversible electrowetting and trapping of charge: model and experiments," Langmuir, vol. 15, no. 20, pp. 6616-6620, 1999.

[84] H. Moon, S. K. Cho, R. L. Garrell, and C. J. Kim, "Low voltage electrowetting-on-dielectric," Journal of Applied Physics, vol. 92, no. 7, pp. 4080-4087, 2002.

[85] A. R. Wheeler, H. Moon, C. A. Bird et al., "Digital microfluidics with in-line sample purification for proteomics analyses with MALDI-MS," Analytical Chemistry, vol. 77, no. 2, pp. 534-540, 2005. 
[86] S. K. Cho, H. Moon, and C. J. Kim, "Creating, transporting, cutting, and merging liquid droplets by electrowetting-based actuation for digital microfluidic circuits," Journal of Microelectromechanical Systems, vol. 12, no. 1, pp. 70-80, 2003.

[87] M. Abdelgawad, S. L. S. Freire, H. Yang, and A. R. Wheeler, "All-terrain droplet actuation," Lab on a Chip, vol. 8, no. 5, pp. 672-677, 2008.

[88] M. Vallet, B. Berge, and L. Vovelle, "Electrowetting of water and aqueous solutions on poly(ethylene terephthalate) insulating films," Polymer, vol. 37, no. 12, pp. 2465-2470, 1996.

[89] S. N. Pei, J. K. Valley, S. L. Neale, A. Jamshidi, H. Y. Hsu, and M. C. Wu, "Light-actuated digital microfluidics for largescale, parallel manipulation of arbitrarily sized droplets," in Proceedings of the 23rd IEEE International Conference on Micro Electro Mechanical Systems (MEMS '10), pp. 252-255, January 2010.

[90] T. B. Jones, Electromechanics of Particles, Cambridge University Press, 1995.

[91] H. Pohl, Dielectrophoresis, Cambridge University Press, Cambridge, UK, 1978.

[92] P. Y. Chiou, A. T. Ohta, and M. C. Wu, "Massively parallel manipulation of single cells and microparticles using optical images," Nature, vol. 436, no. 7049, pp. 370-372, 2005. 

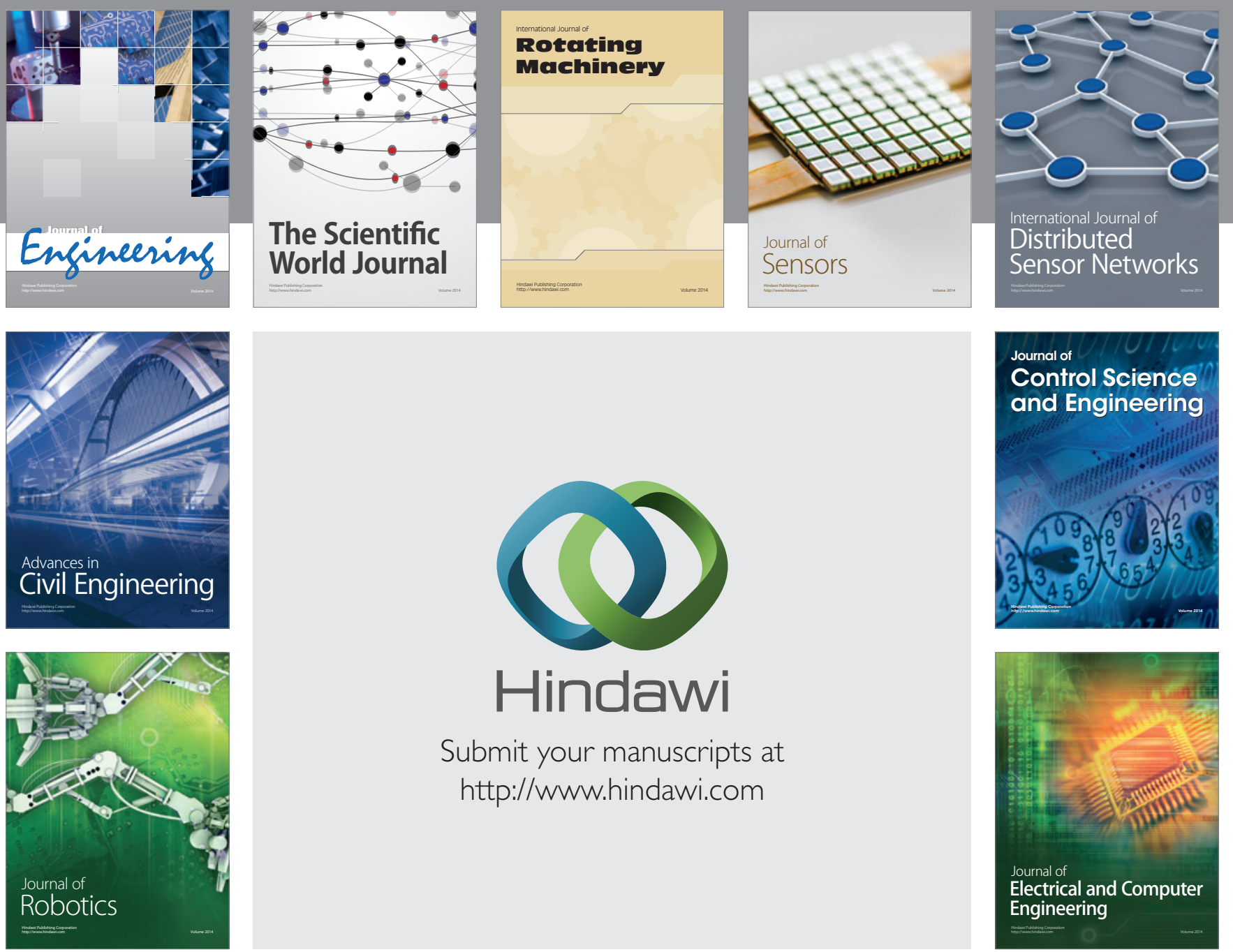

Submit your manuscripts at

http://www.hindawi.com
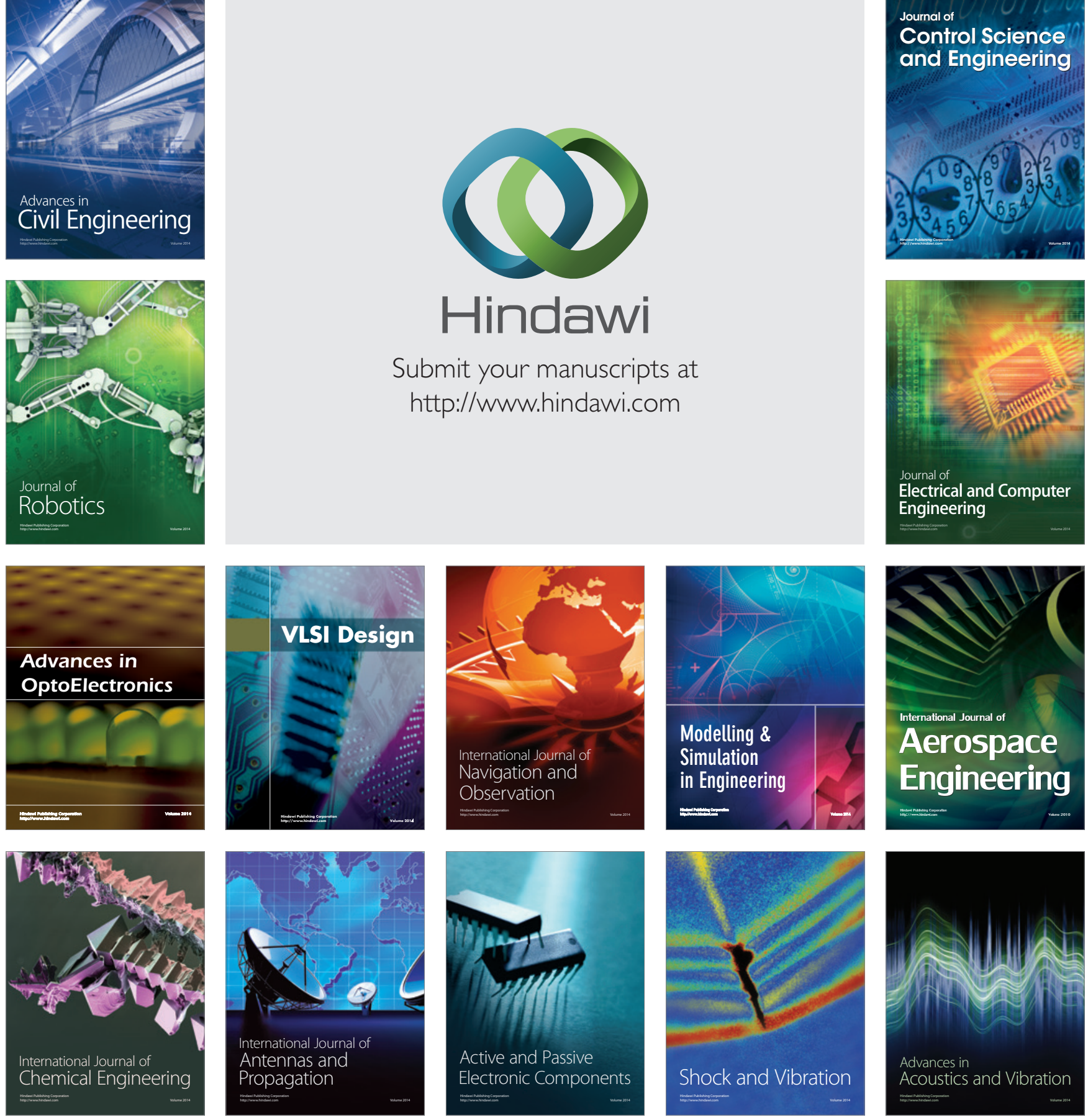